\title{
Neues System zur Prüfung von Fahrleitungen und Stromabnehmern
}

\author{
Tilman Grabowski, Marko Erbacher, Manfred Deutzer
}

\section{Einleitung}

Eine Optimierung des Zusammenwirkens von Fahrleitung, Stromabnehmer und Schleifleisten unter Berücksichtigung der Eigenheiten des Fahrzeuges und des Gleises ist sehr schwierig. Alle Komponenten unterliegen einer ständigen Veränderung. Die Eigenschaften der Fahrleitung hängen unter anderem von der Temperatur ab. Die Stromabnehmer und Schleifleisten verschleißen. Die Federung in den Fahrzeugen altert.

Trotz der ständigen Veränderung des Systems muß ein ausreichend gutes Zusammenwirken der Fahrleitung mit dem Stromabnehmer und den Stromabnehmerschleifleisten gewährleistet werden.

An der Optimierung des Systems arbeitet die Firma Deutzer Technische Kohle GmbH (DTK) seit einigen Jahren. Von DTK wurden zahlreiche Prifmethoden entwikkelt, mit denen eine immer bessere Einstellung des Systems möglich wurde.

Mit diesen Prïfmethoden wurde bei über 100 Verkehrsunternehmen in Europa das System Fahrleitung - Stromabnehmer - Schleifleiste untersucht und verbessert. Zusätzlich zu den Untersuchungen der Fahrleitung und der Schleifleisten wurden von DTK und dem Ingenieurbüro Wilpsbäumer neue Stromabnehmer entwickelt, die sich besonders gut in das Stromentnahmesystem integrieren lassen. Hierzu wurden auch zahlreiche Untersuchungen an den unterschiedlichen Wippenausführungen vorgenommen. Aus allen diesen Arbeiten konnte DTK Erkenntnisse gewinnen, die es ermöglichten ein neues Meßsystem aufzubauen, daß erhebliche Vorteile gegenuiber dem bisher verwendeten Meßsystem besitzt.

Das neue DTK-System dient der dynamischen Überprüfung des Zustandes und des Betriebsverhaltens von Stromentnahmesystemen oberleitungsgebundener Schienenfahrzeuge. Das hier beschriebene Prüfverfahren stellt eine Verbesserung aller bisher entwickelten DTK-Systeme dar; die zur Zick-Zack- und Höhenlagebestimmung der Fahrleitung, zur Messung der Stöße zwischen Fahrleitung und Schleifleisten und zur Ermittlung der fahrdynamischen Eigenschaften des Stromabnehmers verwendet wurden. Auf der Grundlage der Meßergebnisse mit dem neuen System lassen sich kritische Stellen und Bereiche im Verlauf der Fahrleitung zuverlässig und komfortabel ermitteln. Anschließend können festgestellte Störstellen beseitigt und das Zusammenspiel von Fahrleitung und Stromabnehmer unter Berïcksichtigung der konkreten Streckenbedingungen optimal aufeinander abgestimmt werden. In der Folge sinkt der Wartungs- aufwand am Stromabnehmer und den Oberleitungsanlagen, verringern sich Störungen im täglichen Fahrbetrieb und Stillstandszeiten der Fahrzeuge.

Mit dem Meßsystem können während einer Meßfahrt gleichzeitig folgende Parameter gemessen, dargestellt und aufgezeichnet werden:

- Die Seitenlage der Fahrleitung

- Die Höhenlage der Fahrleitung

- Die Stöße, die in vertikaler und in horizontaler Richtung auf die Schleifleisten wirken (Die Stoßempfindlichkeit kann so eingestellt werden, daß die Laufruhe der Stromabnehmerwippe an der Fahrleitung bestimmt wird.)

- Der elektrische Strom, der über jede Schleifleiste fließt und seine Fließrichtung

- Die Fahrgeschwindigkeit

- Das Verhalten des Stromabnehmers (Es wird mit einer Videokamera aufgenommen und überlagert mit einer grafischen Darstellung der aktuellen Meßdaten auf einem Videofilm aufgezeichnet.)

Soll die Fahrleitungsseitenlage zum Gleis gemessen werden, wird zusätzlich die seitliche Schaukelbewegung des Fahrzeugs mit ermittelt. Eine andere Möglichkeit, die Seitenlage der Fahrleitung zum Gleis zu ermitteln, besteht in der Blockierung der Federung.

Nachfolgend soll das neue DTK-System vorgestellt werden und es werden einige Ergebnisse von Fahrleitungsprüfungen bei unterschiedlichen europäischen Verkehrsunternehmen diskutiert. Das neue System wurde bereits von mehreren Verkehrsunternehmen gekauft.

\section{Beschreibung des neuen DTK-Systems}

Wie bei allen vorherigen DTK-Systemen werden die aufgenommenen Daten vom Stromabnehmer über Lichtwellenleiter in das Fahrzeuginnere geleitet. Somit ist das Fahrleitungspotential und das Potential im Fahrzeug getrennt.

Die Messung der Parameter erfolgt vollautomatisch, gesteuert durch die DTK-Software. In bestimmten Abständen wird an den Meßcontroller der Befehl zur Messauslösung erteilt. Daraufhin veranlaßt dieser die Bestimmung der Fahrdrahtlage, fragt die Daten der Stoßsensoren und des Höhengebers ab und übermittelt alle gesammelten Daten über den Wegcontroller an den Rechner. Dort werden die Meßdaten verarbeitet, auf dem Monitor grafisch dargestellt und in einer Meßdatei gespeichert. 
Der Abstand zwischen zwei Messungen wird durch die einzugebende Datenrate bestimmt. Die Datenrate ist die Anzahl der Messungen pro gefahrenem Streckenmeter oder pro Zeiteinheit. Der Befehl zur Meßauslösung kann entweder

- in Abhängigkeit von der tatsächlich zurüickgelegten Wegstrecke

oder

- in bestimmten konstanten Zeitabständen erzeugt werden.

Bei Dienstleistungen durch DTK kann das Meßsystem in ca. zwei Stunden auf jedes beliebige Fahrzeug mit Stromabnehmer aufgebaut werden. Dann wird das vom Auftraggeber vorgegebene Netz befahren. Nach der Prüfung wird das System in maximal zwei Stunden abgebaut. Danach steht das Fahrzeug wieder für einen Linieneinsatz zur Verfuigung. Bei einem geringen Fahrzeugbestand kann das DTK System auf Fahrzeuge des normalen Linienverkehrs aufgebaut werden. Im Fahrzeug befindet sich dann nur ein PC bzw. Laptop.

Sofort nach der Prüfung erhält der Auftraggeber eine Kurzeinschätzung. Innerhalb von 4 Wochen wird dann ein umfangreicher Untersuchungsbericht und die Videoaufzeichnung geliefert. Neben den Ergebnissen werden in dem Bericht sofort durchzuführende Maßnahmen empfohlen.

DTK wurde von Auftraggebern bestätigt, daß die Störungen nach den Prüfungen und der anschließenden Fehlerbeseitigung drastisch zuruickgegangen sind.

\subsection{Fahrdrahtseitenlage}

Die Fahrdrahtseitenlage wird mittels einer speziell von DTK entwickelten und gebauten Meßleiste bestimmt. Die Meßleiste wird für den Meßbetrieb unmittelbar vor bzw. hinter einer der Schleifleisten montiert. Auf der Oberseite der Meßleiste sind in einem Abstand von 2 $\mathrm{cm} 60$ Stuick Infrarot-Sendedioden und eine gleiche Anzahl Empfängerdioden verteilt.

Hat die Fahrleitung von vornherein größere oder kleinere Zick-Zack-Lagewerte, kann das Meßlineal um jeweils vier Sender-Empfängerpaare verlängert oder verkürzt werden.

Nach dem Signal zur Meßauslösung aktiviert der Meßcontroller die Elektronik, die sich ebenfalls in der Meßleiste befindet. Die Infrarotsensoren strahlen einen kurzen Lichtimpuls ab. Dieser wird von der Unterseite der Fahrleitung reflektiert. Befindet sich die Fahrleitung zwischen zwei Empfängerdioden, erhalten beide ein Signal. Die Signale der Empfängerdioden werden von der Elektronik ausgewertet und entsprechend aufbereitet.

Die Bestimmung der Seitenlage erfolgt berührungslos mit einer Genauigkeit von ca. $1 \mathrm{~cm}$. Die Seitenlage einer oder mehrerer nebeneinander liegender Fahrleitungen kann gleichzeitig bestimmt werden.

\subsection{Fahrdrahthöhenlage}

Die Messung der Fahrdrahthöhenlage erfolgt mit einem Weg/Winkel-Wandler mit angebautem Drehwinkelauf- nehmer. Für die Bestimmung der Höhenlage ist vor der Meßfahrt eine Eichung vorzunehmen. Die Höhenlagebestimmung erfolgt mit einer Genauigkeit von $0,5 \mathrm{~cm}$. Im Meßwert ist der Anhub der Fahrleitung durch den Stromabnehmer enthalten.

\subsection{Stöße auf die Schleifleiste}

Die Stoßaufnahme ist von DTK so empfindlich eingestellt, daß die geringste Laufunruhe der Schleifleisten ermittelt werden kann.

Mit dem neuen System können in einer Prüffahrt die Zick-Zack-Lage, die Höhenlage, die Stöße auf die Schleifleisten in horizontaler und vertikaler Richtung, die fahrdynamischen Eigenschaften des Stromabnehmers, die Geschwindigkeit, der Ort und markante Streckenpunkte aufgezeichnet werden. Die Aufzeichnungsrate (Zahl der Datensätze pro Meter oder pro Zeiteinheit) kann beliebig eingestellt werden.

\subsection{Messung des über die Schleifleisten übertrage- nen elektrischen Stroms}

Zu den Stößen können auch die Anpreßkräfte der Schleifleisten gegen die Fahrleitung oder der über die Schleifleisten übertragene elektrische Strom aufgezeichnet werden. Bei der Strommessung kann auch die Stromrichtung aufgezeichnet werden.

\subsection{Programmbeschreibung}

Die DTK-Software arbeitet unter Windows 95, Windows 98 und Windows NT. Somit besteht die Möglichkeit, die vorhandene Software wie Druckertreiber, Datenbanken, Kalkulationsprogramme, Grafikprogramme, Bildverarbeitungsprogramme usw., sowie die vorhandenen Peripheriegeräte zu nutzen. Eine mögliche Anwendung wird im Abschnitt 3.2 beschrieben.

Nachfolgendes Bild 1 zeigt den Computermonitor während der Aufzeichnung der Daten. Für eine schnelle Einarbeitung besitzt die Software den Menüpunkt „Simulation". Hiermit hat der Anwender die Möglichkeit zu üben. Er kann sich schon vor der ersten Prüfung mit dem Programm vertraut machen.

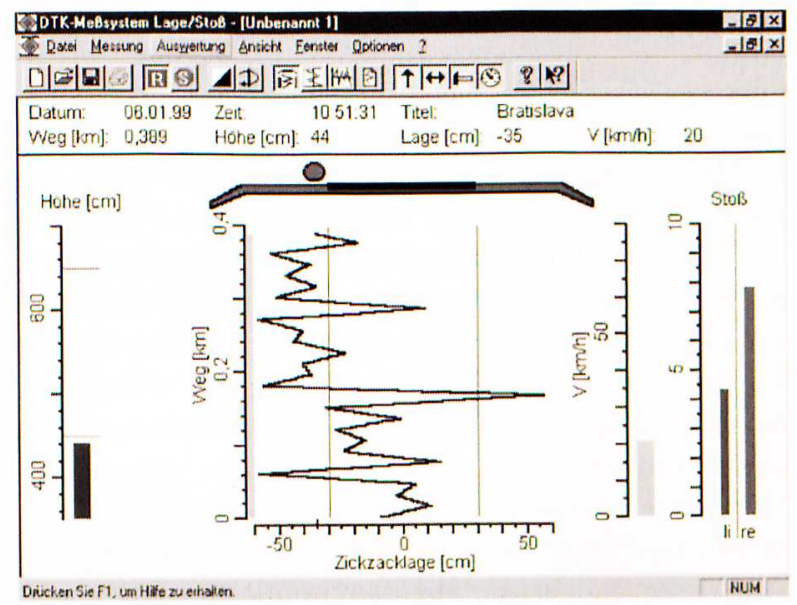

Bild 1: Computermonitor während der Datenaufnahme 
Auf der linken Bildseite ist die Fahrdrahthöhe, in der Mitte die Fahrdrahtseitenlage mit der Abszissenachse „Zickzacklage“ und der Ordinatenachse „Weg“ dargestellt. Daneben befindet sich ein Balkendiagramm für die gefahrene Momentangeschwindigkeit. Rechts außen sind die Diagramme für Stöße in horizontaler und vertikaler Richtung für die zwei Schleifleistenseiten zu sehen. Für die Fahrdrahthöhen- und -seitenlage können vor oder während der Messung Grenzbereiche beliebig eingestellt werden. In der Darstellung liegen die Grenzen bei $450 \mathrm{~cm}$ und $650 \mathrm{~cm}$ (waagerechte rote Striche) bzw. bei $-30 \mathrm{~cm}$ und $+30 \mathrm{~cm}$ (senkrechte rote Linien). Alle Achsen wie Weg, Geschwindigkeit, Zickzacklage und Stoß können beliebig gestreckt oder gestaucht werden.

Über der graphischen Darstellung sind das Datum der Prïfung, die Zeit, der Titel der Prüfung (bei Dienstleistungen meist der Ortsname) sowie die Meßwerte des letzen Datensatzes - die Ortsposition (Weg), die Fahrleitungshöhe, die Seitenlage der Fahrleitung und die Geschwindigkeit dargestellt.

Die Ikons in der oberen Symbolleiste bedeuten der Reihenfolge nach: Neue Meßdatei, Öffnen, Speichern, Drucken, Aufzeichnung starten, Messung unterbrechen, invertierte Bildschirmfarben, Spiegelung der Lageachse, Ansicht Schleifleiste Balken (siehe Bild 1), vertikale Darstellung, horizontale Darstellung, Druckansicht, Höhenlage, Seitenlage, Stoß, Geschwindigkeit, Info und Hilfe.

Bereits während der Prüfung können die Daten von bestimmten Punkten untersucht werden oder es können Punkte zusätzlich markiert werden. Mit der Maustaste können Cursoren gesetzt werden. Die Daten für die gewählten Punkte werden am oberen Bildrand dargestellt. Wird die Taste „z" gedrückt, dann wird der zwischen den Cursoren liegende Bereich auf dem gesamten Koordinatenfeld dargestellt. Mit der Pfeiltaste " und mit „ $\rightarrow$ " gestreckt. Mit der Pfeiltaste „^““ wird das Koordinatenfeld Seitenlage/Weg in Richtung Dateianfang und mit „ $\downarrow “$ in Richtung Dateiende bewegt. Während dieser Untersuchung läuft die Aufzeichnung weiter.

Durch Aktivieren des Ikons „invertierte Bildschirmfarben" wird der Bildschirm für die Videoaufzeichnung vorbereitet. Die weißen Flächen - Bild 1 - werden schwarz und damit durchsichtig, so daß hier das Videobild zu sehen ist. Das Videobild und die Computergrafik werden so iiberlagert auf einer Videokassette aufgezeichnet.

Da die Videokamera entweder in Fahrtrichtung oder entgegen der Fahrtrichtung montiert sein kann, besteht die Möglichkeit, die Seitenlageachse zu spiegeln. Dadurch wird der seitliche Verlauf der Fahrleitung auf dem Computerbild und dem Videobild synchronisiert.

Mit den Unterprogrammen Datei, Messung, Auswertung, Ansicht, Fenster, Optionen und ? können beliebige Einstellungen des Systems gewählt werden.

\subsubsection{Wegerfassung und Wegkorrektur}

Anhand der Wegerfassung und Wegkorrektur soll ein Teil der vielen Möglichkeiten, die die Software bietet, erklärt werden.

Nach der Installation des Systems und dem Starten des Programms kann der vom Fahrzeug bereitgestellte Wegimpuls an das DTK-System angepaßt werden. Hierzu wird das folgende Eingabefenster aufgerufen (Bild 2).

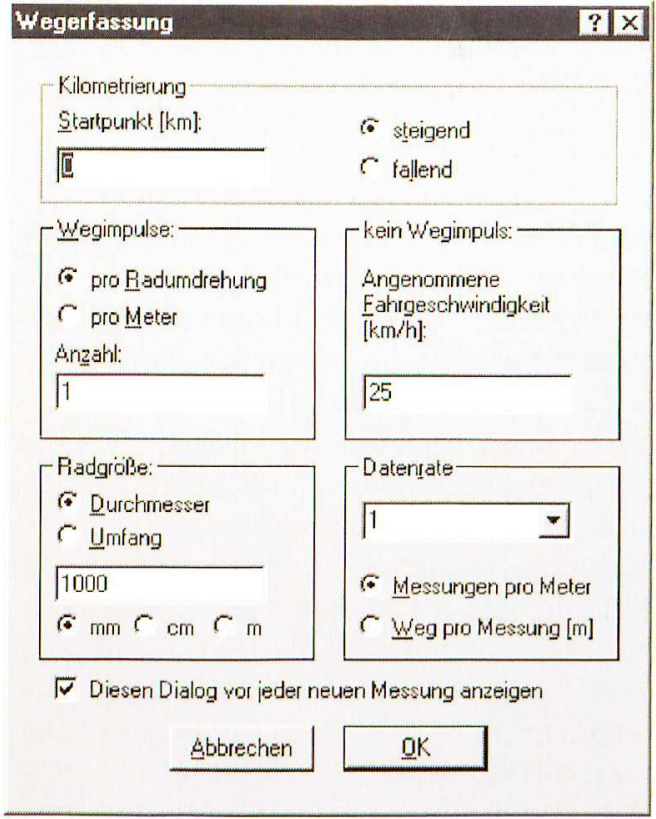

Bild 2: Eingabefenster für die Wegimpulse

In diesem Fenster werden dann folgende Einstellungen gewählt:

Der Startkilometer wird in das obere Eingabefeld eingetragen. Auf der gegenuiberliegenden Seite wird angegeben, ob die Prüfung in Richtung zunehmender Kilometerzahlen erfolgen soll, oder in der entgegengesetzten Richtung. Somit kann das Programm auf eingleisigen Strecken an die vorhandene Kilometrierung angepaßt werden. Das System bietet auch die Möglichkeit, das zu befahrende Liniennetz zu kilometrieren.

Anschließend wird im Feld Wegimpulse ausgewählt, ob sich die vom Fahrzeug bereitgestellten Impulse auf eine Radumdrehung oder auf einen Streckenmeter beziehen. Danach wird die Zahl der Impulse eingegeben. Bei der Angabe pro Radumdrehung wird im Feld Radgröße der Raddurchmesser oder der Umfang eingegeben.

Werden vom Fahrzeug keine Wegimpulse zur Verfügung gestellt, so kann entweder von DTK ein externer Wegimpuls bereitgestellt werden oder die Prüfung wird ohne Wegimpuls durchgeführt. Bei der Prüfung ohne Wegimpuls sollte mit einer gleichmäßigen Prüfgeschwindigkeit gefahren werden. Die Prüfgeschwindigkeit wird im Feld „kein Wegimpuls“ eingetragen.

Im Feld „Datenrate“ wird festgelegt, wieviele Messungen pro Meter durchgefuihrt werden sollen. 


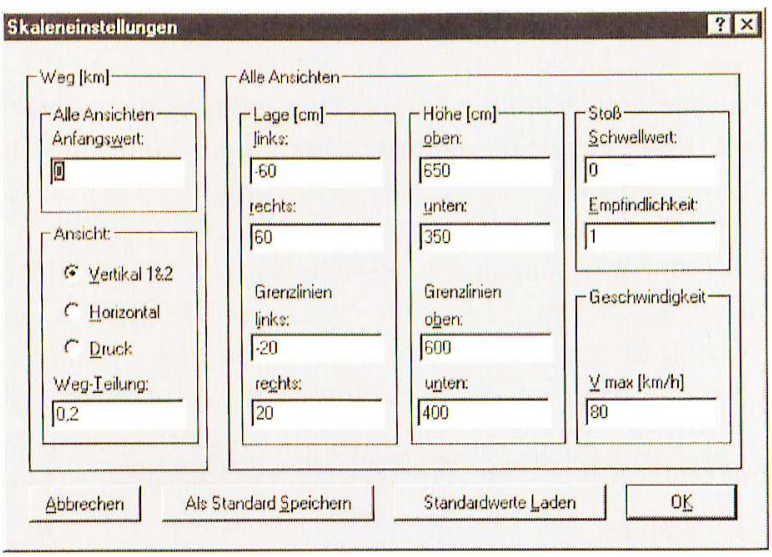

Bild 3: Skaleneinstellungen

Im Bild 3 wird die Skaleneinstellung für die einzelnen Prüfgrößen festgelegt. Weiterhin können hier Grenzbereiche voreingestellt werden. Mit dem Feld „Weg - Teilung“ kann die Unterteilung der Wegachse voreingestellt werden.

Bei ungenauer Angabe der Zahl der Wegimpulse kann es zu Differenzen zwischen der tatsächlichen Streckenlänge und der gemessenen Prüfstrecke kommen. Mit dem im Bild 4 dargestellten Unterprogramm kann die Prïfstreckenlänge nachträglich korrigiert werden. Es können zwei bekannte Werte „Startpunkt, Endpunkt oder Länge der Strecke" eingegeben werden, um sofort den fehlenden Wert berechnen zu lassen. Nach dem Bestätigen mit „OK“ werden die berechneten Daten übernommen und im Diagramm dargestellt. Es besteht auch die Möglichkeit die Länge der Strecke innerhalb von zwei frei gewählten Cursoren beliebig zu strecken oder zu stauchen.

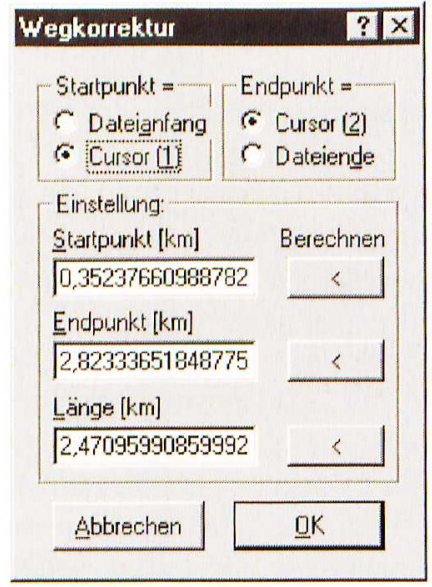

Bild 4: Wegkorrektur

Nachdem die Einstellungen für die Wegaufnahme in der gewünschten Form erfolgt sind, werden sie gespeichert. Bei jedem weiteren Programmaufruf werden diese Voreinstellungen dann verwendet.

\subsubsection{Darstellungsarten}

Die aufgenommenen Daten können in mehreren Formen dargestellt werden. Die Bilder 5, 6, 7 und 8 zeigen die Möglichkeiten der Darstellung in horizontaler Richtung, in vertikaler Richtung und für den Drucker.
In der vertikalen Darstellung im Bild 5 sind die Höhenlage, Zick-Zack-Lage und die Geschwindigkeit dargestellt. An der linken Bildseite befinden sich die Merkmale für die gewählten markanten Streckenpunkte. Die Daten fuir die Cursoren sind über der Grafik dargestellt. Der Cursor 2 wurde an der Position des Fahrleitungswechsels gesetzt. An dieser Streckenposition wurde von dem System die einlaufende Fahrleitung mit -15 $\mathrm{cm}$ und die auslaufende Fahrleitung mit $3 \mathrm{~cm}$ registriert.

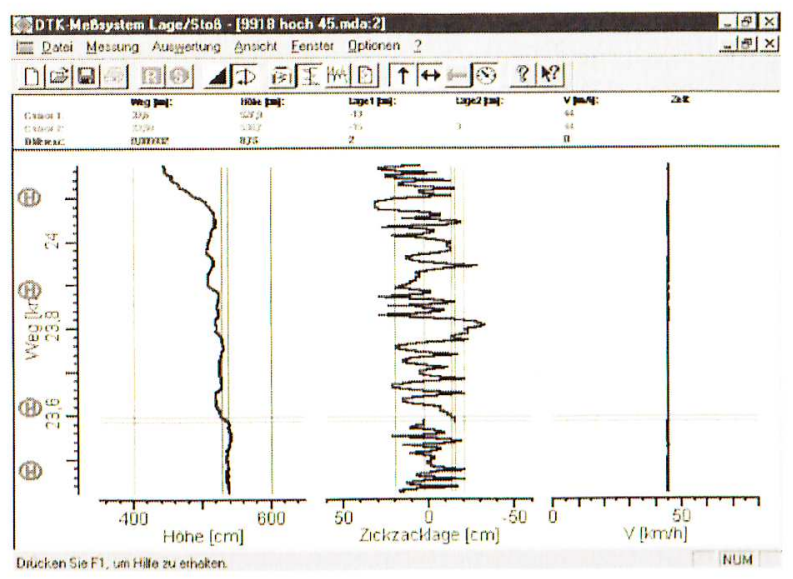

Bild 5: Vertikale Darstellung der Höhe, Seitenlage, Geschwindigkeit und markanten Punkte für eine Prüfstrecke

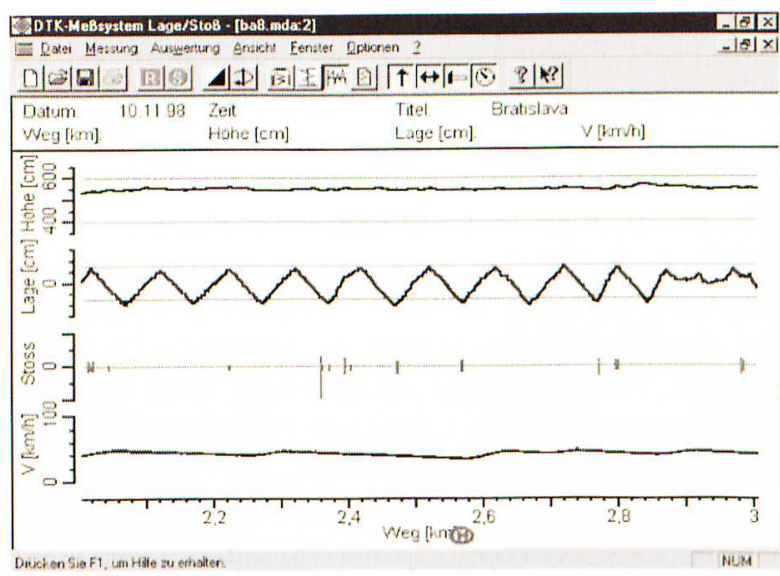

Bild 6: Horizontale Darstellung der Höhenlage, Seitenlage, Geschwindigkeit, Stöße horizontal und vertikal

Das Bild 6 zeigt die horizontale Darstellung für einen Streckenabschnitt von einem Kilometer. Bei dieser Prüfung in Bratislava wurden die Höhenlage, die Seitenlage, die Stöße auf beiden Schleifleistenseiten in horizontaler und vertikaler Richtung, die Geschwindigkeit und markante Punkte aufgenommen.

Bei der Ausgabe der Daten auf einem Drucker hat der Anwender die Möglichkeit, die Druckansicht, den Bereich vom Cursor 1 bis zum Cursor 2, den Bereich vom Dateianfang bis zum Cursor 2, den Bereich von Cursor 1 bis zum Dateiende, die gesamte Datei oder die gesamte Datei mit Deckblatt ausdrucken zu lassen (Bild 7). Auf dem Deckblatt werden dann u.a. die wichtigen Dateidaten wie Dateiname, Streckenname, Datum, Ort, Zeit und alle eingetragenen Informationen zur Prüfung, zum Fahrzeug und zur Strecke dargestellt. 


\section{Druckbereich \\ C Druckansicht: \\ (- Cursor 1 bis Cursor 2 \\ C Dateianfang bis Cursor (2) \\ C Cursor (1) bis Dateiende \\ C Gesarnte Datei \\ $\Gamma$ mit Deckblatt}

x

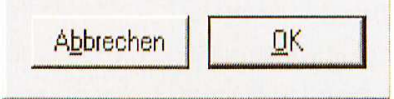

Bild 7: Bestimmung der Druckerausgabe

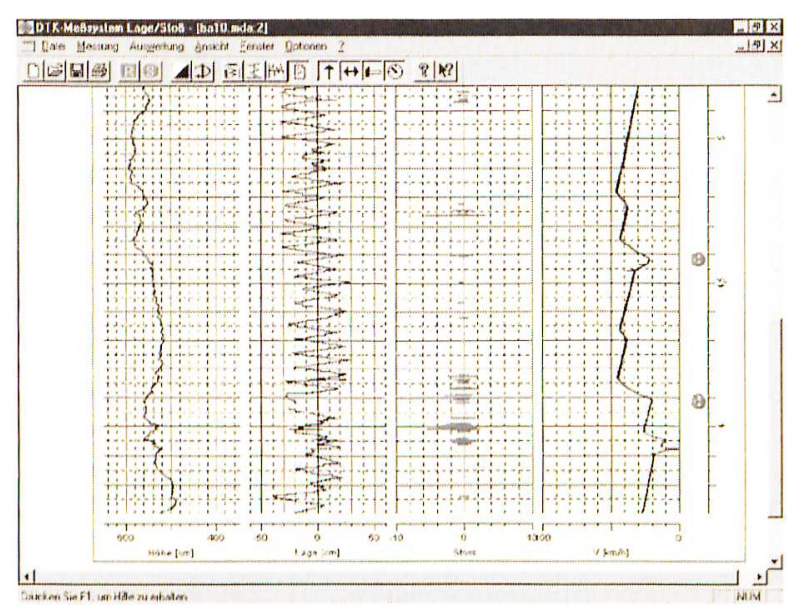

Bild 8: Druck-Darstellung Höhe, Seitenlage, Geschwindigkeit, Stöße und markante Streckenpunkte

Das Bild 8 zeigt eine Darstellung der Druckansicht für die Höhenlage, die Seitenlage, die Stöße zwischen der Fahrleitung und den Schleifleisten und die Prüfgeschwindigkeit.

Das Bild 9 zeigt einen vorbereiteten Stromabnehmer zur Aufnahme der Seitenlage, der Höhenlage, der fahrdynamischen Werte des Stromabnehmers sowie der Stöße zwischen den Schleifleisten und der Fahrleitung.

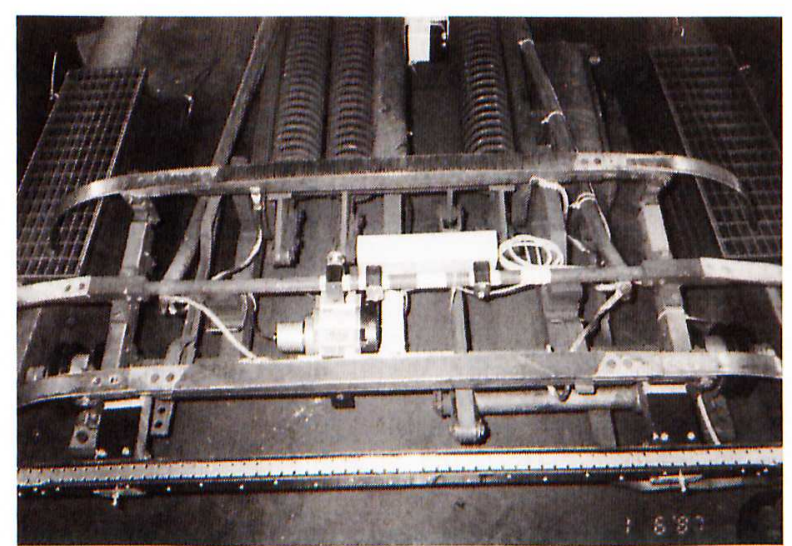

Bild 9: Stromabnehmer mit DTK-Meßsystem

Die während der Prüfung aufgezeichneten Meßdaten werden vom Controller am Stromabnehmer aufgenommen und aufbereitet. Über Lichtwellenleiter gelangen die Daten potentialgetrennt zur Weiterverarbeitung zum Rechner im Fahrzeug.
In den Bildern 10 bis 13 sind einige Fahrzeuge dargestellt, mit denen Fahrleitungsuntersuchungen mit dem beschriebenen neuen DTK-System durchgeführt wurden. Die Fahrzeuge waren zum Teil normale Linienfahrzeuge oder spezielle Meßfahrzeuge.

Es besteht auch die Möglichkeit, die Untersuchungen auf einem Zweiwegefahrzeug mit Stromabnehmer durchzuführen. Bei der Verwendung von Zweiwegefahrzeugen ohne Stromabnehmer kann dieser von DTK zur Verfuigung gestellt werden.
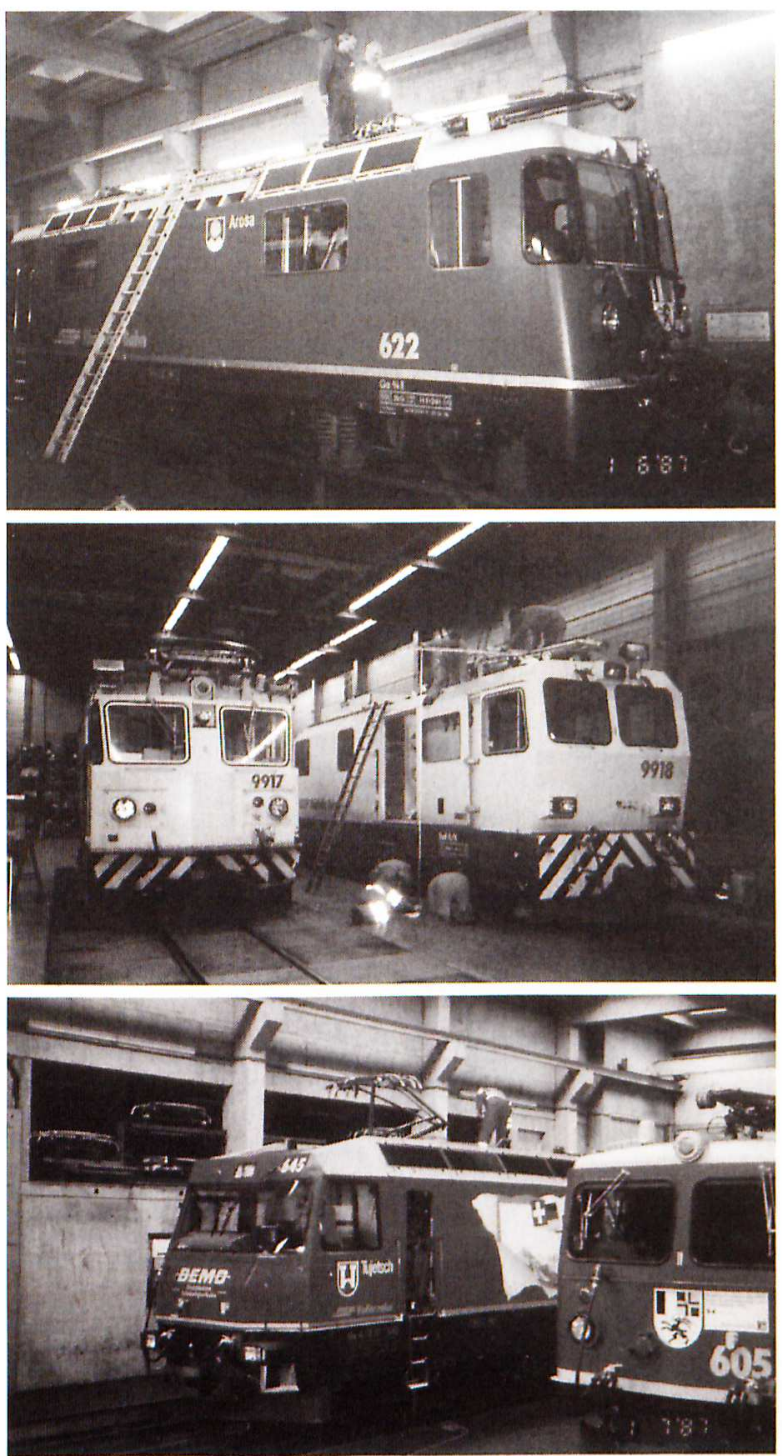

Bild 10a, 10b und 10c: Fünf verschiedene Fahrzeuge der RhB für vergleichende Untersuchungen

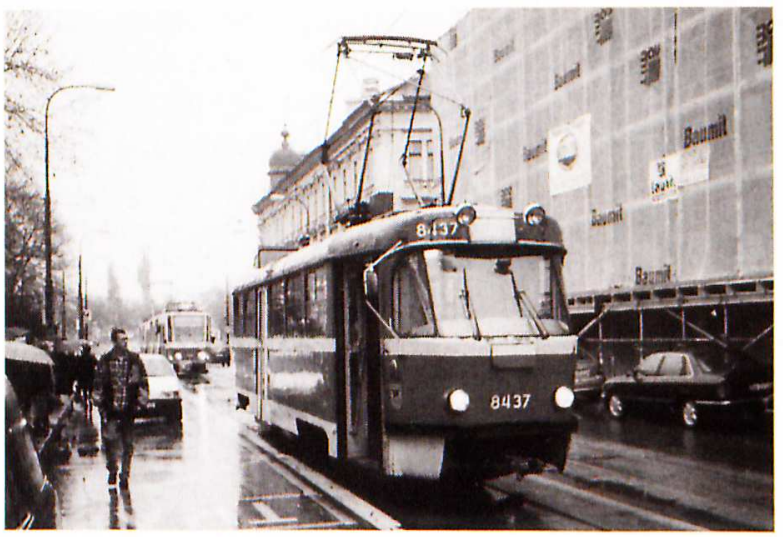

Bild 11: Meßwagen der Verkehrsbetriebe Bratislava 


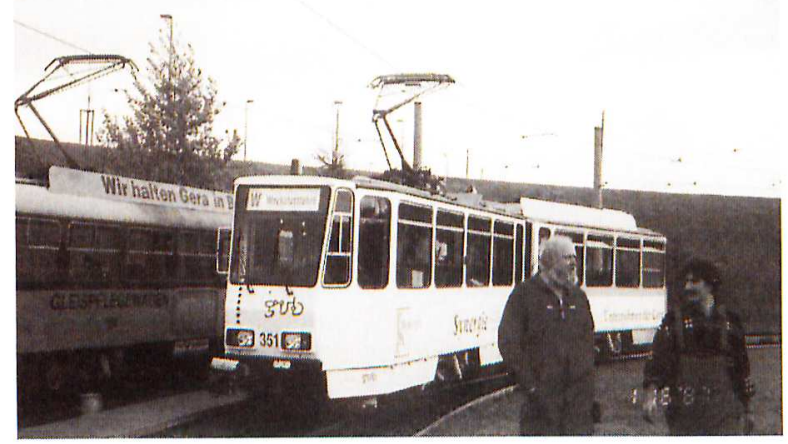

Bild 12: Linienfahrzeug mit Einholmstromabnehmer

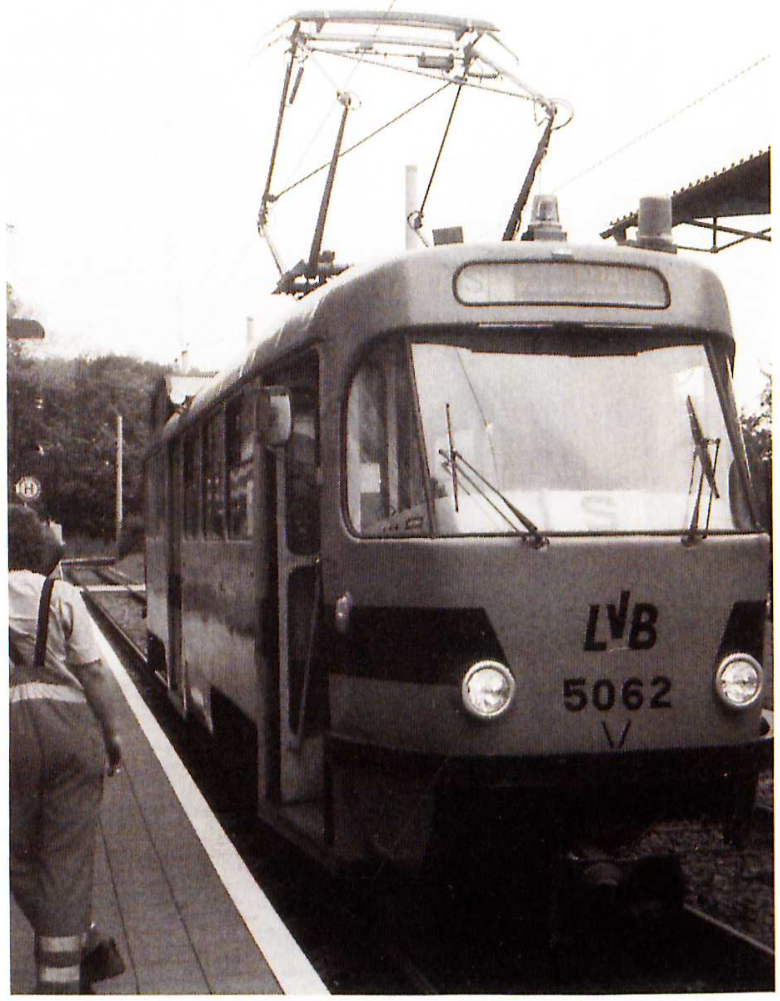

Bild 13: Meßwagen mit Scherenstromabnehmer

Mit dem beschriebenen neuen DTK-System wurden bereits zahlreiche Untersuchungen in mehreren europäischen Ländern durchgeführt. Bei der Durchführung von Dienstleistungen erhält der Auftraggeber einen umfangreichen Untersuchungsbericht, der die Problemstellen auflistet und die erforderlichen Maßnahmen zur Lösung der Probleme beschreibt. Zu dem Untersuchungsbericht gehört ein Videofilm, der das Zusammenspiel von Fahrleitung und Schleifleisten mit den vom Computer grafisch aufbereiteten Meßdaten zeigt.

\section{Auswertungen}

Mit dem neuen Meßsystem Seitenlage, Höhenlage, Stoß, Fahrdynamik, Geschwindigkeit und Ort ist DTK in der Lage das Zusammenwirken der Fahrleitung mit dem
Stromabnehmer zu optimieren. Auf besonderen Wunsch kann die Anpreßkraft der Fahrleitung gegen die Schleifleisten und der über jede Schleifleiste übertragene elektrische Strom zusätzlich ermittelt werden. Bei dem Einsatz von Schopperfahrzeugen kann neben der Strombelastung der Schleifleisten auch die Stromrichtung bestimmt werden.

Mit dem neuen System können die bisher bekannten Auswertungen, wie Fahrleitungsbereiche ohne ausreichenden Zick-Zack, Überschreitung der Außenlagewerte, zu große Höhenänderung, einseitige Aufhängung der Fahrleitung, Verteilungskurven für die Lage usw. bestimmt werden 1,2,3,4. Durch die gleichzeitige Messung aller Größen in einer Prüffahrt, die Aufnahme der Stöße in horizontaler und vertikaler Richtung, sowie die Aufnahme von geringsten Lauf-unruhen ergeben sich weitere Möglichkeiten. Das neue System wurde so gestaltet, daß keine aufwendige Eichung des Systems mehr erforderlich ist.

\subsection{Kinematik des Stromabnehmers}

Für den einwandfreien Lauf von Stromabnehmern ist unter anderem entscheidend, wie schnell und präzise diese einer Höhenänderung des Fahrdrahtes folgen können. Dies gilt sowohl für das Absenken der Stromabnehmer vor einer Brückendurchfahrt, wie auch für das Anheben nach einer Brïckendurchfahrt. Bei derartigen Höhenänderungen ändert sich immer auch die Anpreßkraft der Schleifleisten gegen die Fahrleitung. Eine Änderung der Anpreßkraft wirkt sich jedoch meist negativ auf die Stromübertragung aus. Die Folge ist Bügelfeuer und somit erhöhter elektrischer und mechanischer Verschleiß der Fahrleitung und der Schleifleisten.

Nachfolgend einige Ausschnitte aus Untersuchungsberichten, um die Möglichkeiten zu beschreiben, die das System bietet.

Bei einer Untersuchung des Systems StromabnehmerFahrleitung-Schleifleiste-Fahrzeug in Bratislava wurde das DTK-System Stoß, Zick-Zack, Höhe, Geschwindigkeit, Ort, markante Streckenpunkte und Fahrdynamik eingesetzt. Das System wurde auf ein Fahrzeug mit einem Scherenstromabnehmer aufgebaut (Bild 11). Innerhalb von 4 Stunden wurde das gesamte Streckennetz von $70 \mathrm{~km}$ geprïft.

Aus der Untersuchung wurden mehrere markante Streckenabschnitte ermittelt, die das Zusammenwirken des Stromabnehmers mit der Fahrleitung beschreiben. In den Streckenabschnitten steigt die Fahrleitungshöhe jeweils über einem kurzen Streckenabschnitt. Diese Abschnitte sind Ausfahrten aus Brücken und Tunneln.

Die nachfolgenden Bilder 14 und 15 zeigen eine mögliche Art der Darstellung mit dem DTK-System. Es werden die Fahrleitungshöhe, die Stöße/Fahrdynamik der Stromabnehmerwippe, die Fahrgeschwindigkeit und der Weg dargestellt. 


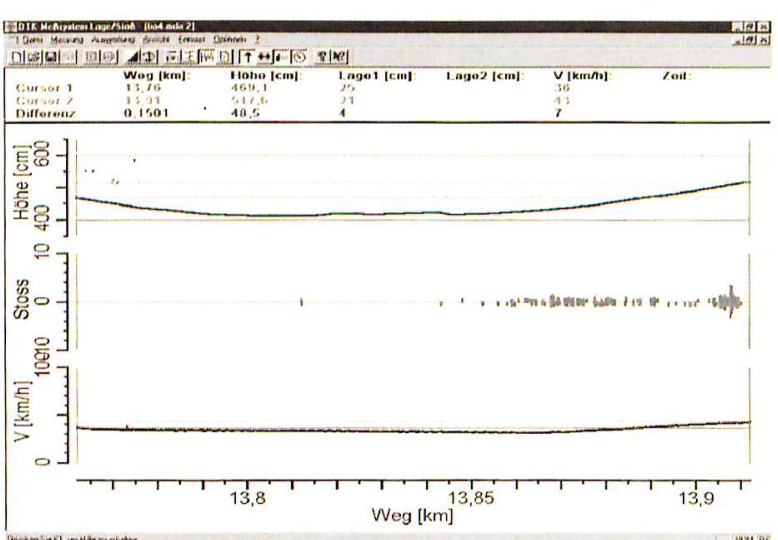

Bild 14: Brückendurchfahrt auf der Linie Dubravka - Jesenskeho - Krizna Posen am Kilometer 13,75 bis 13,93

Im Bild 14 wurde im Bereich 13,810 km bis 13, $850 \mathrm{~km}$ eine Brüicke durchfahren.

Im folgenden wird der Abschnitt in der horizontalen Darstellung gezeigt und die Höhenänderung des Stromabnehmers berechnet bei der Brïckenausfahrt mit einer definierten Geschwindigkeit.

Im Bereich nach der Brïcke, von der Wegposition 13,86 $\mathrm{km}$ bis $13,91 \mathrm{~km}(0,050 \mathrm{~km})$, nimmt die Höhe von 422,8 $\mathrm{cm}$ auf $511,2 \mathrm{~cm}(0,8833 \mathrm{~m}) \mathrm{zu}$. Der Wert für die Höhenänderung $(0,8833 \mathrm{~m} / 50,83 \mathrm{~m})$ liegt bei $1,738 \mathrm{~cm} / \mathrm{Fahr}$ meter. Die Fahrgeschwindigkeit wird von $31 \mathrm{~km} / \mathrm{h}$ auf $42 \mathrm{~km} / \mathrm{h}$ vergrößert. In diesem Bereich tritt eine sehr starke Laufunruhe zwischen Fahrleitung und Schleifleisten auf.

Bei einer durchschnittlichen Fahrgeschwindigkeit von $36,5 \mathrm{~km} / \mathrm{h}(10,14 \mathrm{~m} / \mathrm{s})$ in diesem kritischen Bereich ergibt das eine Höhenänderung von $17,623 \mathrm{~cm} / \mathrm{s}$. Der Stromabnehmer muß innerhalb einer Sekunde einer Höhenänderung von $17,6 \mathrm{~cm}$ folgen und gleichzeitig die geforderte Anpreßkraft von $8 \mathrm{kp}$ einhalten. Da die Trägheit des Stromabnehmers zu groß ist, und er dieser Aufwärtsbewegung nicht folgen kann, kommt es zur Störung der Laufruhe.

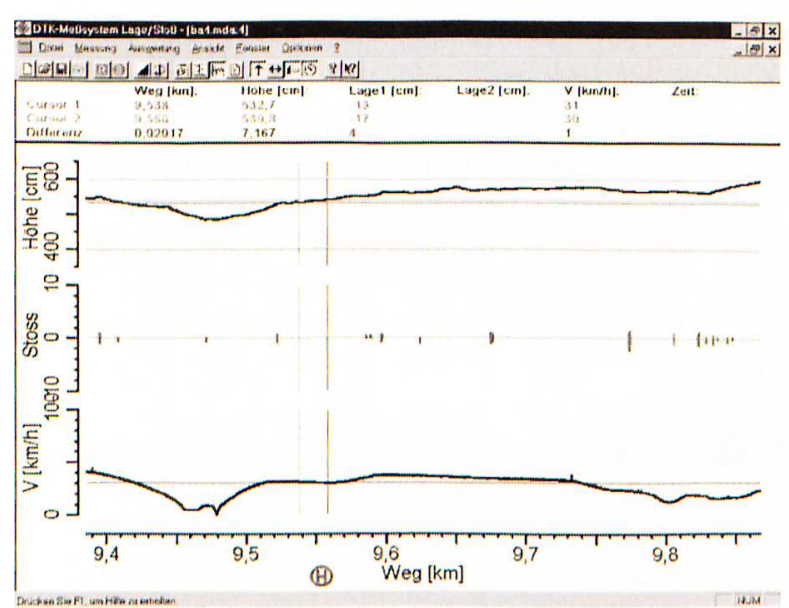

Bild 15: Brückenausfahrt auf der Linie Dubravka - Jesenskeho - Krizna Posen beim Kilometer 9, 4 bis 9,85

Betrachtet man den Abschnitt der größten Laufunruhe von 20 Metern - zwischen den Cursoren Bild 14 - erhält man einen Höhenunterschied von $41,42 \mathrm{~cm}$. Bei einer durchschnittlichen Fahrgeschwindigkeit von 40 $\mathrm{km} / \mathrm{h}(11,11 \mathrm{~m} / \mathrm{s})$ benötigt das Fahrzeug ca. 2 Sekunden um den Abschnitt von $20 \mathrm{~m}$ zu durchfahren. In den 2 Sekunden muß der Stromabnehmer somit den Höhenunterschied von 41,42 cm ausgleichen.

Bei der gleichen Prüfung an einer anderen Streckenposition (Bild 15) wird die Fahrdrahthöhe von 485,5 $\mathrm{cm}$ auf $575,5 \mathrm{~cm}(0,9 \mathrm{~m})$ vergrößert. Die Höhenänderung erfolgt auf einem Streckenabschnitt von $9,481 \mathrm{~km}$ bis $9,648 \mathrm{~km}(0,1678 \mathrm{~km})$. Die Höhenänderung an dieser Stelle $(0,9 / 167,8)$ beträgt 0,536 $\mathrm{cm} /$ Fahrmeter. Die mittlere Geschwindigkeit liegt bei $22 \mathrm{~km} / \mathrm{h}(6,111 \mathrm{~m} / \mathrm{s})$. Es ergibt sich eine Höhenänderung von $0,536 \mathrm{~cm} / \mathrm{m} \times 6,111 \mathrm{~m} / \mathrm{s}=3,375 \mathrm{~cm} /$ s. Der Stromabnehmer muß in diesem Bereich eine Höhenänderung von $3,375 \mathrm{~cm} / \mathrm{s}$ ausgleichen. Diese Änderung kann von dem Stromabnehmer ohne Kontaktverschlechterung vorgenommen werden.

Der Vergleich der untersuchten Streckenabschnitte zeigt, daß der verwendete Stromabnehmer im normalen Fahrbetrieb einer Höhenänderung von 3,375 cm/s ausreichend gut folgen kann. Bei einer Höhenänderung von $17,623 \mathrm{~cm} / \mathrm{s}$ kann der Stromabnehmer nicht mehr ausreichend schnell dem Fahrleitungsverlauf folgen. Die Kontaktpreßkraft zwischen den Schleifleisten und der Fahrleitung sinkt. Es treten partielle Kontaktunterbrechungen auf. Die Laufruhe zwischen den Schleifleisten und der Fahrleitung ist gestört. Partielle Lichtbögen führen zum erhöhten Verschleiß der Fahrleitung und der Schleifkohle. Von DTK wird in einem solchen Fall vorgeschlagen

- entweder die Höhenänderung über einen längeren Streckenabschnitt vorzunehmen

- oder die Fahrgeschwindigkeit zu verringern

- oder die Kinematik des Stromabnehmers zu verbessern

- oder die Anpreßkraft der Schleifleisten zu verändern

- oder ....

Bei anderen Verkehrsunternehmen wurde das gleiche Problem mit anderen Stromabnehmern untersucht. Auch hier zeigten die Ergebnisse die gleiche Tendenz. Der ermittelte Bereich für den Übergang von einer ausreichenden Laufruhe zu einem gestörten Kontaktverhalten unterscheidet sich bei den unterschiedlichen Stromabnehmertypen.

Die Stromabnehmer unterschieden sich untereinander durch ihren Aufbau. Einige Stromabnehmer hatten einzeln abgefederte Schleifleisten, andere hatten Rahmenwippen oder das Stromabnehmergestell war zusätzlich gedämpft. Eine Dämpfung des Stromabnehmergestells wirkt der Fähigkeit, dem sich ändernden Höhenverlauf der Fahrleitung zu folgen stets entgegen. Oft ist diese Dämpfung jedoch erforderlich, um ruhig laufende Antriebssysteme zu erhalten.

\subsection{Einfluß des Fahrzeuges, des Gleises und des Stromabnehmers auf die Lage der Fahrleitung}

Bei einer Untersuchung bei der Rhätischen Bahn in der Schweiz sollte mit dem DTK-System „Seitenlage und Höhenlage“ die Lage der Kontaktstelle Fahrleitung 
Stromabnehmer bei unterschiedlichen Fahrzeugtypen ermittelt werden. Hierzu wurde das System nacheinander auf fünf verschiedene Fahrzeuge aufgebaut und ein definierter Streckęnabschnitt mit unterschiedlichen Geschwindigkeiten (bis zu $102 \mathrm{~km} / \mathrm{h}$ ) in Bergaufund Bergabrichtung befahren. Bei vier Fahrzeugen konnte das DTK-System direkt an den vorhandenen Wegimpuls im Fahrzeug angeschlossen und geeicht werden. Im fünften Fahrzeug gab es keinen Wegimpuls. Deswegen wurde mit diesem Fahrzeug mit einer definierten Prïfgeschwindigkeit gefahren. Nach den Untersuchungen konnte mit Hilfe der im DTK Programm vorgesehenen Wegkorrektur die gemessene Streckenlänge korrigiert werden. Die Diagramme der Meßfahrten wurden auf Folien ausgedruckt. Durch Aufeinanderlegen der Folien konnten die Unterschiede in der Seitenlage in Abhängigkeit von der Fahrgeschwindigkeit, der Seitenstabilität der Stromabnehmer oder vom Fahrzeugtyp ermittelt werden. Die Ergebnisse sind in dem DTK-Untersuchungsbericht Nr. 144/98 RhB vom 24.11.1998 enthalten. Im Nachgang zu den Untersuchungen wurde von der RhB bei DTK ein Meßsystem gekauft und auf das Fahrzeug Nr. 9918 installiert.

Das Bild 16 a und b zeigt eine Gegenüberstellung für einen bestimmten Streckenabschnitt der Prüffahrten mit dem Fahrzeug Nr. 9918 - 45 km/h und mit dem Fahrzeug Nr. $605-50 \mathrm{~km} / \mathrm{h}$.

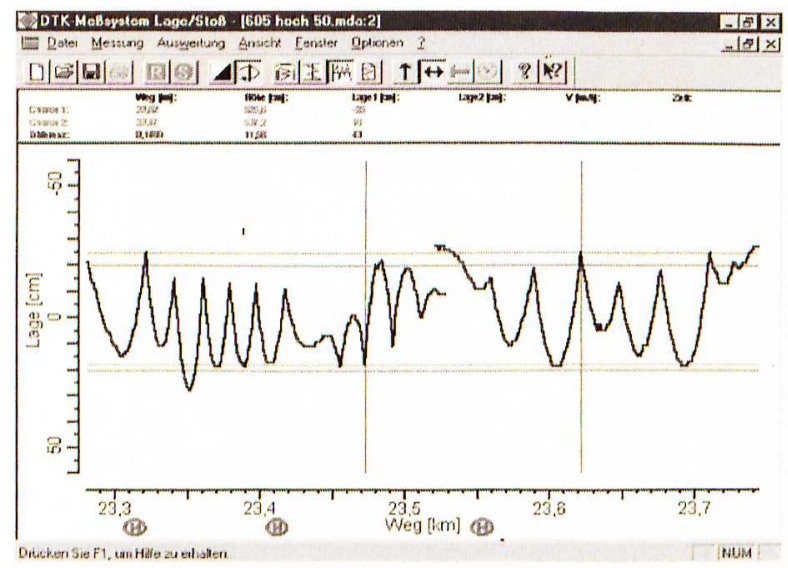

Bild 16a: Ausschnitt aus der Prüfung mit dem Fahrzeug 9918 - 45 km/h und

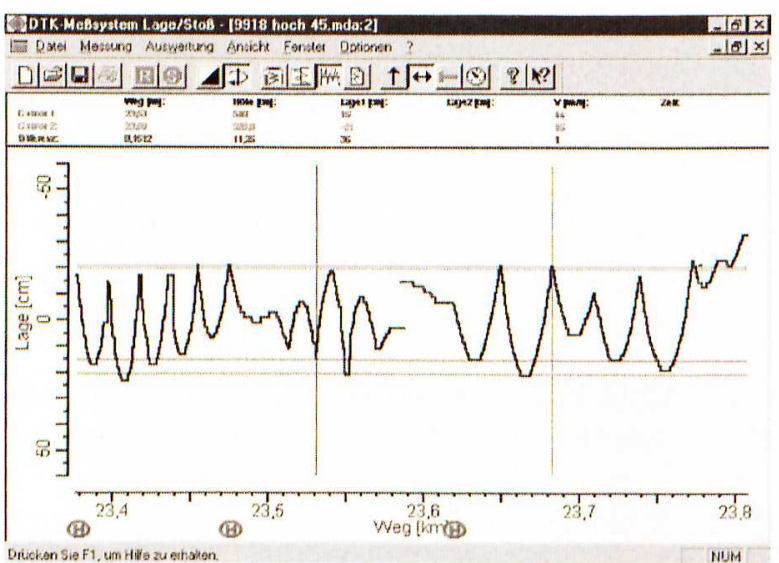

Bild 16b: mit dem Fahrzeug 605 - 50 km/h

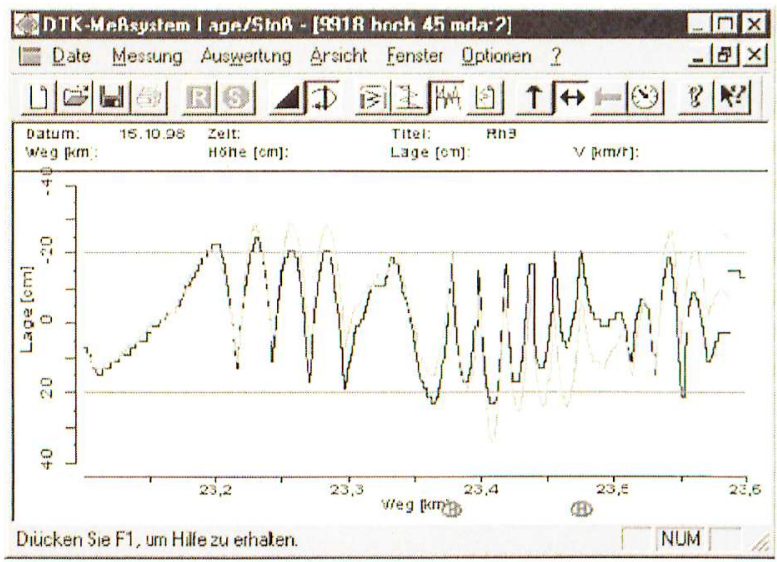

Bild 17: Überlagerung der Seitenlage für zwei Meßfahrten mit unterschiedlichen Fahrzeugen

An den gleichen Wendepunkten (Cursoren) liegen die Seitenlagewerte für die Fahrt mit dem Fahrzeug 605 (oben) bei $-25 \mathrm{~cm}$ und bei $+18 \mathrm{~cm}$ und mit dem Fahrzeug 9918 (unten) bei $-21 \mathrm{~cm}$ und bei $+15 \mathrm{~cm}$. Die Seitenlage der Kontaktpunkte zwischen der Fahrleitung und den Schleifleisten dieser beiden Fahrzeuge unterscheiden sich bei den gewählten zwei Positionen um 4 und $3 \mathrm{~cm}$. Bei dieser Untersuchung wurden Lageunterschiede zwischen anderen Fahrzeugen, bei gleicher Geschwindigkeit von bis zu $18 \mathrm{~cm}$ ermittelt.

Da die DTK-Software auf der Windowsoberfläche arbeitet, besteht die Möglichkeit mit Hilfe von Bildverarbeitungsprogrammen die aufgenommenen Kurven fuir einen Vergleich direkt übereinander zu legen.

Einen solchen Vergleich zeigt das Bild 17. Hier wurde die Zick-Zack-Lage der Fahrleitung für den Streckenabschnitt von $23,1 \mathrm{~km}$ bis $23,6 \mathrm{~km}$ ausgeschnitten und auf das Seitenlagediagramm für den gleichen Streckenabschnitt eingeführt. Die dunkle Kurve des Fahrleitungs-Zick-Zacks stellt die Lage des Kontaktpunktes Fahrleitung-Schleifleiste für das Fahrzeug Nr. 9918 bei einer Prüfgeschwindigkeit von 45 $\mathrm{km} / \mathrm{h}$ dar, die helle Kurve zeigt sie für das Fahrzeug Nr. 645 bei $50 \mathrm{~km} / \mathrm{h}$.

Es konnte festgestellt werden, daß die Lageunterschiede außer von der Fahrgeschwindigkeit auch vom Bogenradius des Gleises entscheidend beeinflußt werden.

\subsection{Stromabnehmer mit unzureichender Seiten- stabilität}

In diesem Beispiel (Bild 18) ist der Zick-Zack-Verlauf des Fahrdrahtes von einer zweiten Schwingung uiberlagert. Die Besonderheit hier besteht jedoch darin, daß die Periodendauer dieser Schwingung deutlich kleiner ist und die Ursache für diese Schwingung nicht allein in der Schwankung des Fahrzeugs bestehen kann. Dieses Erscheinungsbild weist deutlich auf eine sehr geringe Seitenstabilität des verwendeten Stromabnehmers hin. Der Stromabnehmer wurde nach der Prüfung einer eingehenden Inspektion unterzogen. Es zeigte sich, daß die Lager des Stromabnehmers verschlissen waren. 


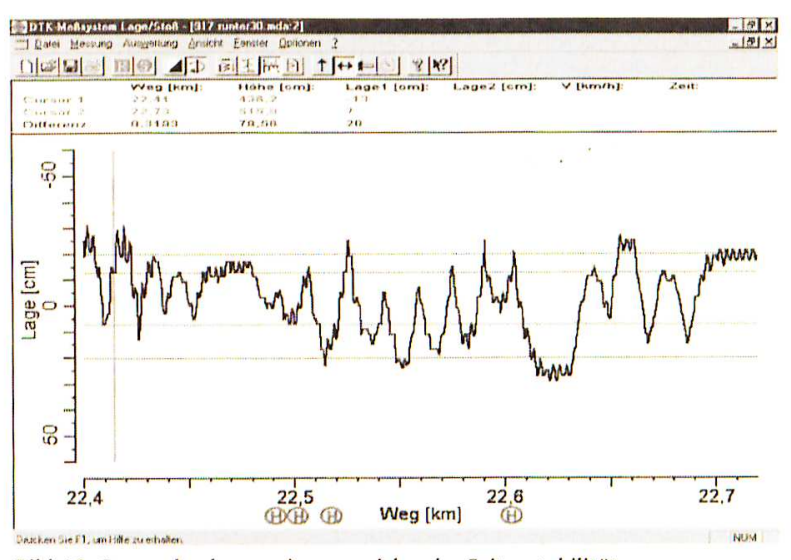

Bild 18: Stromabnehmer mit unzureichender Seitenstabilität

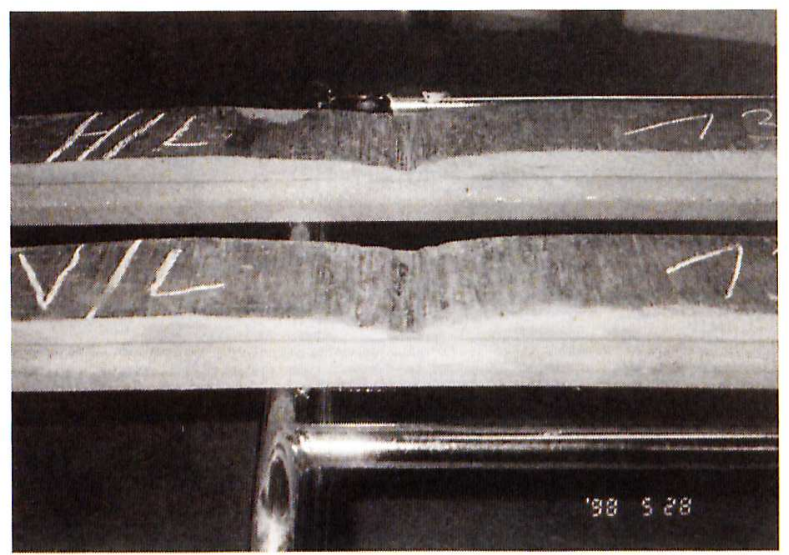

Bild 19: Schleifkohle mit eingeschliffener Querrille.

\subsection{Untersuchung von Querrillen in der Schleifkohle}

In einem anderen Fall wurde das DTK System genutzt um die Ursache für die Entstehung von Querrillen in der Schleifkohle zu ermitteln. Bild 19 zeigt die geschädigten Schleifleisten mit Querrille. Mit dem DTK System wurde das Streckennetz befahren und festgestellt, daß die Fahrleitung in einem bestimmten Streckenabschnitt vorwiegend in dem geschädigten Bereich der Schleifleiste liegt (Bild 20). Die Verteilungskurve für die Kontaktstelle zeigt an der Position $-30 \mathrm{~cm}$ bis $-35 \mathrm{~cm}$ eine deutliche Anhäufung. Bei der Prüfstreckenlänge von 4,762 km befand sich die Fahrleitung sehr häufig in dem geschädigten Bereich. Rechnet man die Teilstuicken zusammen, dann erhält man bei $-32 \mathrm{~cm}$ bis $-34 \mathrm{~cm}$ eine Streckenlänge von $220 \mathrm{~m}$ und bei $-30 \mathrm{~cm}$ bis $-32 \mathrm{~cm}$

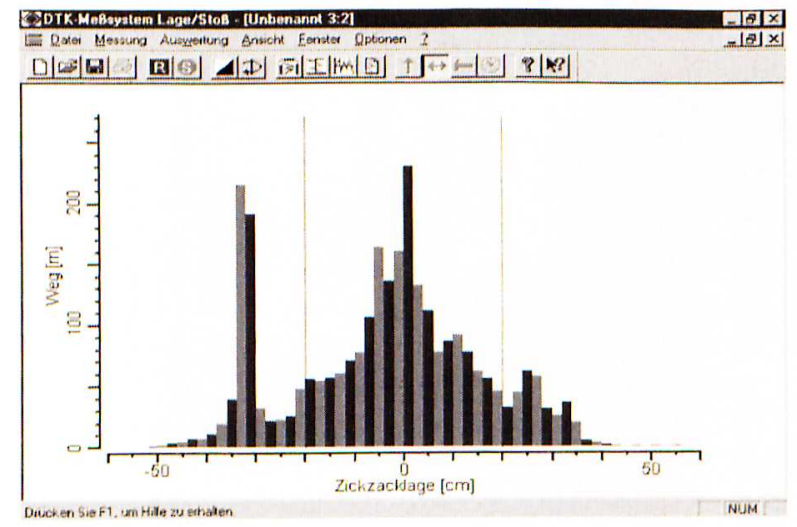

Bild 20: Häufigkeitsverteilung ca. $180 \mathrm{~m}$. In den benachbarten Bereichen dagegen nur ca. $40 \mathrm{~m}$ bis $50 \mathrm{~m}$.

Durch einen häufigen Aufenthalt der Fahrleitung in dem selben Bereich der Schleifleiste oder durch elektrischthermische Überlastung der Schleifleiste entsteht leicht eine Querrille. Anschließend wird die Fahrleitung über längere Streckenabschnitte in der Querrille gehalten. Hierdurch wird der Kohlekörper zusätzlich elektrisch und mechanisch überlastet.

Nach der Prïfung konnten sofort Maßnahmen getroffen werden, um zuküinftig die Bildung von Querrillen in der Schleifkohle zu vermeiden.

Aufgrund der bekannten Probleme bei Fahrzeugen mit Stromruickspeisung wurde das System von DTK so erweitert, daß auch der iiber die Schleifleisten entnommene bzw. in das Netz eingespeiste Strom aufgezeichnet werden kann.

\subsection{Stoßstellen an Fahrdrahtaufhängungen und Streckentrennern}

Die grafische Darstellung Bild 6 zeigt an den Streckenpositionen 2,02 km, 2,22 km, 2,38 km, 2,48 km, 2,58 $\mathrm{km}, 2,78 \mathrm{~km}$ und 2,8 km Stoßstellen. Wenn man die Seitenlage an diesen Streckenpositionen betrachtet, dann fällt auf, daß die Stöße immer an äußeren Wendepunkten, den Fahrdrahtabspannungen, auftritt.

Das Bild 21 zeigt eine Strecke, bei der an der Position $6,569 \mathrm{~km}$ ein sehr großer Stoß auftritt. Die Fahrleitungsseitenlage befindet sich hier an der Position „2 cm“, daß heißt, in der Mitte der Schleifleiste. Aus der gleichzeitig durchgefuihrten Videoaufzeichnung ist zu erkennen, daß dieser Stoß von einem Streckentrenner verursacht wurde. Die Prüfgeschwindigkeit an dieser Stelle betrug $32 \mathrm{~km} / \mathrm{h}$ und die Fahrleitungshöhe lag bei 6,569 Meter.

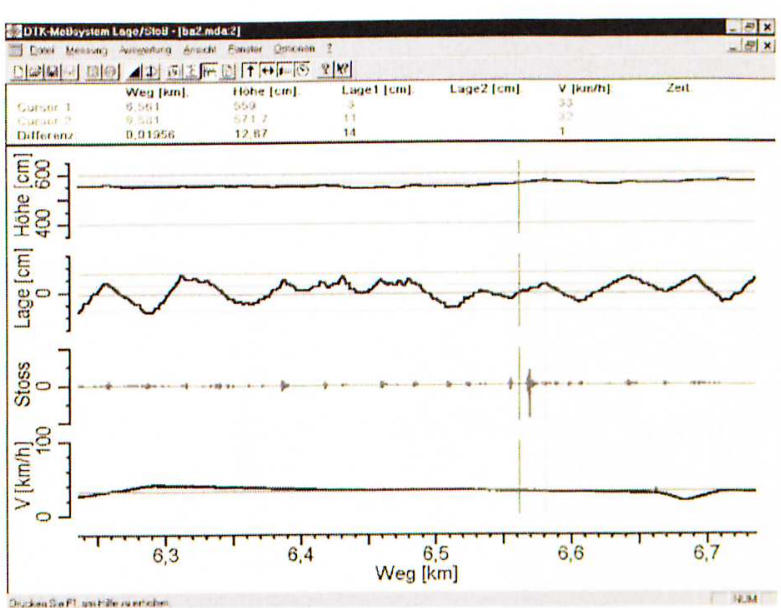

Bild 21: Stoßstellen von Fahrdrahtabspannungen und von einem Streckentrenner

Bei Untersuchungen bei den Wiener Verkehrsbetrieben wurde mit dem DTK-System die optimale Einbaulage von Streckentrennern ermittelt. Bei den Arbeiten konnten die Stöße zwischen Streckentrennern und Schleifleiste minimiert werden. Die Wiener Verkehrsbetriebe haben nach den Untersuchungen das DTK System Stoß gekauft. 
Das DTK-Meßsystem wurde so gestaltet, daß es besonders gut auf die jeweilige Aufgabenstellung angepaßt werden kann. Bei besonderen Aufgabenstellungen kann das System entsprechend erweitert werden. Ein Beispiel hierfür war der Einsatz des DTK-Systems zur Messung des Lichtraumes im Rad-Schiene-Bereich.

\section{Zusammenfassung}

Das neue DTK System zur Pruifung des Systems Fahrleitung-Stromabnehmer-Schleifleiste steht dem Nutzer seit ca. einem Jahr zur Verfügung. Es wird von DTK für Dienstleistungen genutzt oder an Endabnehmer direkt verkauft. Das System liefert, neben den bekannten Aussagen zur Fahrleitungslage und zu den Stößen auch Informationen zur Kinematik des Stromabnehmers und zum Fahrzeug sowie zum Zusammenwirken der einzelnen Komponenten. Die Prüfungsergebnisse werden während der Prüffahrt auf einem Monitor bzw. TV-Gerät dargestellt, so daß der Nutzer bereits während der Prüfung die fehlerbehafteten Stellen sieht und sofort entsprechende Maßnahmen einleiten kann. Durch ein nochmaliges Durchfahren der Strecke nach der Korrektur der Fehlerstellen kann der Erfolg der getroffenen Maßnahmen sofort überprüft werden. Eine nachträgliche aufwendige Auswertung entfällt.

Nach der Durchfuihrung von Dienstleistungen durch DTK kann der Auftraggeber auf Wunsch zusätzlich zum Untersuchungsbericht eine Datendiskette erhalten. Er hat dadurch die Möglichkeit zusätzliche gewünschte Auswertungen in kürzester Zeit auf seinem PC selbst durchzufiihren.

Somit ist das DTK System hervorragend für Fahrleitungsinspektionen und zur Fehlersuche geeignet. Das Meßsystem kann nach einem geringen Anpassungsaufwand auch zur Lösung anderer Probleme genutzt werden.

\section{Literatur}

[1] Deutzer Technische Kohle GmbH - DER NAHVERKEHR, Heft 3/96, Seite 50-53

[2] Stimming/Behrend/Richter/Deutzer - DER NAHVERKEHR, Heft 9/97, Seite 64-69

[3] Deutzer Technische Kohle GmbH - VERKEHR UND TECHNIK, Heft 4/98, Seite 139-140

[4] Deutzer Technische Kohle GmbH - VERKEHR UND TECHNIK, Heft 7/98, Seite 282

\section{Autoren}

\section{Dipl. Ing. Tilman Grabowski}

Dipl. Ing. Marko Erbacher

Dipl. Chem. Manfred Deutzer (Geschäftsführer)

Deutzer Technische Kohle GmbH (DTK)

Bahnhofstraße, Gebäude 19

15745 Wildau

Tel. (0 33 75) 552000

Fax (0 33 75) 552001

FuTel. (01 71) 8187303

E-Mail: info@deutzer.de

http://www.deutzer.de

Marzahner Promenade 28

12679 Berlin

Tel. (0 30) 93554230

Fax (0 30) 93554231 